\title{
Netzwerke in der Pädiatrie aus Sicht der Klinischen Pädiatrie
}

\section{Networking in Pediatrics and the Journal's View}

U. Göbel ${ }^{1}$,

U. Kontny ${ }^{2}$,

L. Gortner ${ }^{3}$

T. Klingebiel ${ }^{4}$

${ }^{1}$ Herausgeber und Bandredakteur

${ }^{2}$ Bandredakteur

${ }^{3}$ Herausgeber

${ }^{4} \mathrm{GPOH}$-Vorsitzender

\section{Bibliografie}

DOI 10.1055/s-0029-1220719 Klin Padiatr 2009; 221: 131-133

(c) Georg Thieme Verlag KG

Stuttgart · New York

ISSN 0300-8630

Korrespondenzadresse

Prof. em. Dr. U. Göbel

Heinrich-Heine-Universität

Düsseldorf

Moorenstraße 5

40225 Düsseldorf

Goebel@

med.uni-duesseldorf.de
Mit ihren Schwerpunktbänden dokumentiert die Gesellschaft für Pädiatrische Onkologie und Hämatologie $(\mathrm{GPOH})$ seit 1977 regelmäßig ihre überregionalen Kooperationen und ihre fachliche Interdisziplinarität bei der Diagnostik und Behandlung von gut- und bösartigen Blut- und Tumorerkrankungen. Darüber hinaus hat sie mit ihren Schwerpunktbänden 36 (Klinische Pädiatrie Heft 6/2007) und 38 (Klinische Pädiatrie Heft $6 / 2008$ ) die Immunologie in gleichwichtiger Weise wie die Hämatologie und Onkologie berücksichtigt, nicht nur um auf Gemeinsamkeiten aufmerksam zu machen, sondern auch um Synergien zu mobilisieren. Der hier vorliegende Schwerpunktband 39 hat diesmal neben den hämatologischen und onkologischen Themen als Arbeits- und Forschungsbereich die Hämostasiologie mit einer Übersichtsarbeit und vier Fallberichten berücksichtigt. Auch wenn die Immunologie und die Hämostasiologie innerhalb der Kinder- und Jugendmedizin ihre eigenständigen Profile besitzen, sind sie als Querschnittfächer eng mit der Hämatologie und Onkologie verbunden.

In der Übersicht berichten Röseler und Mitarbeiter über die Septine, eine neue Familie von GTPbindenden Proteinen (s.S. 150-155). Diese spielen bei grundlegenden zellulären Prozessen wie der Exozytose von Vesikeln oder Zytokinese eine wichtige Rolle. So sind die Septine bei der Degranulation von Thrombozyten, aber auch bei der Sekretion von Neurotransmittern beteiligt. In der Onkogenese können Septine den Fusionspartner bei einem MLL-Rearrangement stellen, aber auch als Tumorsuppressorgene agieren.

In einem klassischen Fallbericht beschreiben Weinspach und Mitarbeiter (s.S. 167-171) den Algorithmus bei der Diagnosestellung von schweren Blutungsereignissen bei vorher klinisch unauffälligen Kindern. Anlässlich einer intrakranialen Blutung wurde bei einem weiblichen Kleinkind eine schwere Hämophilie A entdeckt, die pathogenetisch auf ein klinisch noch asymptomatisches Turner-Syndrom und eine Neumutation in dem einen X-Chromosom zurückgeführt wird; weiterhin werden die bisher bekannten anderen pathogenetischen Wege zu dieser sehr seltenen Konstellation der Hämophilie bei einem Mädchen gelistet, um schneller zur richtigen Diagnose zu gelangen. Mit einem eindrucksvollen Bildbericht stellen Bernstädt und Mitarbeiter (s.S. 172-173) Pseudotumoren bei zwei Patienten mit einer mittelschweren Hämophilie vor. Diese als Folge von Einblutungen vor allem im Bereich der Muskulatur entstehenden Tumoren kommen für gewöhnlich nur bei schwerer Hämophilie vor und werfen Fragen insbesondere bezüglich der Abwägung zwischen konservativem und operativem Management auf. In einem weiteren Pictorial Essay weisen Walter und Mitarbeiter (s.S. 174-175) darauf hin, dass bei einer systemischen Heparinisierung im Rahmen eines thrombotischen Ereignisses auch die Thrombusgröße im Verlauf regelmäßig zu kontrollieren und bei Größenzunahme an eine Heparin-induzierte und antikörpervermittelte Thrombozytopenie II (HIT) zu denken ist. In einer Short Communication stellen Mauz-Koerholz und Mitarbeiter (s.S. 180) einen 12-jähriger Jungen mit passagerer inkompletter Tetraplegie vor, bei dem im Rahmen der Thrombophiliediagnostik ein heterozygoter Protein-S-Mangel (Aktivität 45\%, Konzentration $40 \%$ ) festgestellt wurde; die genetische Untersuchung erbrachte eine neue Missensemutation in Exon 10 des Protein-S-Gens: in Position 865 wurde ein Basenaustausch $(C>T)$ nachgewiesen, der mit einem Austausch der Aminosäuren Arg289Trp (R289W) verbunden ist.

Ziel dieser Präsentationen ist auch, gleichartige Krankheitsverläufe in klinischen Registern zu sammeln, um den Stellenwert derartiger Mutationen oder Antikörperproduktionen bei Kindern mit akuten Krankheitssituationen für die Diagnostik und Therapie besser beurteilen zu können. Darüber hinaus sind die Fallberichte Beispiele für den hohen Grad der Vernetzung innerhalb der Pädiatrie und Belege für die erforderliche und auch verfügbare Methodenvielfalt, um zu Diagnosen unter Berücksichtigung der Pathogenese zu gelangen.

Während Onkologie, Hämatologie und Hämostasiologie Bereiche sind, die sich eng berühren, hat die Informatik, der der erste Übersichtsartikel gewidmet ist, noch nicht den ihr gebührenden Platz in unserem klinischen Alltag erlangt. Die Informatik hat in den letzten Jahren unsere diagnostischen Möglichkeiten revolutioniert, wie dies beispielhaft die moderne Schnittbilddiagnostik belegt, ist jedoch bisher nicht direkt in therapeutische Entscheidungen integriert. Die Berührung so unterschiedlicher Wissensgebiete wie die klinische Onkologie und die Informatik birgt jedoch ein ungemeines Potential für innovative Lösungen. Basierend auf der zunehmenden Leistungsfähigkeit von Computern und den Fortschritten in der Systemanalyse stellen Graf und Mitautoren (s.S. 141-149) in ihrer Übersicht ein computergestütztes System zur Vorhersage des Therapieansprechens von Tumoren vor. Damit ein solcher Oncosimulator funktioniert, bedarf es der Integration vielfältiger klinischer und biolo- 
gischer Daten sowie pharmakologischer Parameter, die zum einen vom Patienten, zum anderen aus der Literatur stammen. Am Beispiel eines Kindes mit Nephroblastom werden der simulierte und der beobachtete Therapieverlauf vergleichend dargestellt; das Ziel ist, durch eine solche Computersimulation vor Therapiestart diejenigen Patienten identifizieren zu können, die auf eine präoperative Chemotherapie nicht ansprechen würden. Solche Patienten könnten dann direkt der Operation zugeführt und womöglich eine ineffektive Chemotherapie erspart werden. Damit ein solches System klinisch zur Anwendung kommen kann, sind aber Validierung und Selbstlernfunktion unabdingbare Vorraussetzungen.

Bei den Originalarbeiten berichtet Peeters mit der LESS-Arbeitsgruppe (s.S. 156-161) über die prospektiv gemessene gesundheitsbezogene Lebensqualität bei 96 chemotherapeutisch behandelten ALL-Patienten, die in 15 deutschen Studienzentren von 1997-2003 behandelt wurden. Mit Hilfe eines Eltern- und eines Patientenfragebogens wurde die Lebensqualität an drei definierten Zeitpunkten erfasst: bis zu 2 Wochen nach Diagnosestellung, nach Beendigung der Reinduktionstherapie und nach Abschluss der Dauertherapie. Verglichen mit einer allgemeinen Stichprobe aus der Gesamtbevölkerung wurde in dieser Patientengruppe in allen Testkomponenten eine signifikant niedrigere Lebensqualität gemessen, die sich jedoch im zeitlichen Verlauf sowohl aus Sicht der Eltern als auch der Kinder signifikant verbesserte. Derartige prospektive Kohortenstudien sind aufwändig, aber sehr viel aussagekräftiger als retrospektive Querschnittuntersuchungen, was besonders für konsekutive Therapieoptimierungsstudien unter dem Blickwinkel der primären Rehabilitation von großem Wert ist. Andere Aspekte enthält die Arbeit von Chen und Mitautoren (s.S. 162-166), in der die Therapie begleitende Untersuchungen epikritisch ausgewertet werden: Hier wurde bei 168 Kindern und Jugendlichen, die bei unterschiedlichen onkologischen Diagnosen eine Therapie mit Anthrazyklinen erhielten, mittels regelmäßig erfolgenden EKG- und Echokardiografieuntersuchungen nach kardialen Komplikationen Ausschau gehalten. Zum einen traten bei einer mittleren Nachbeobachtungszeit von 5 Jahren nur bei 4 Patienten kardiale Komplikationen auf; nur eine davon wurde aber durch die Routineuntersuchung erkannt. Diese Auswertung belegt, dass die ergriffenen Maßnahmen die Kardiotoxizität der aktuellen Therapieoptimierungsprotokolle reduziert hat, wirft aber die Frage nach dem Benefit und der Art regelmäßig durchgeführter kardiologischer Untersuchungen unter Anthrazyklintherapie auf, um die Patienten mit verminderter Anthrazyklintoleranz frühzeitig zu erkennen.

Bei den klassischen Fallberichten unterstreichen Escobar und Mitautoren (s.S. 176-178) einmal mehr, hinter welch unterschiedlicher Maskierung sich die akute lymphatische Leukämie verbergen kann. Im dargestellten Fall wurde eine ALL durch Nierenbiopsie bei einem Kind mit unklarer akuter Niereninsuffizienz diagnostiziert, die sich unter der nicht-onkologischen Therapie erstmal zurückbildete; das Blutbild war mit Ausnahme einer leichten Anämie unauffällig und deshalb nicht diagnostisch relevant.

Maligne Hodentumoren sind bei jungen Männern die häufigste maligne Erkrankung und gelten seit Einführung der Cisplatinbasierten Chemotherapie durch Einhorn und Donohue [7]. als in hohem Maße kurabel. Mit zwei Fallberichten werden Hochrisikopatienten präsentiert, die auf der Grundlage einer standardisierten Therapie individualisierten Therapiestrategien zugeführt wurden (s.S. 136-140 und 179). Mit seinem Gastkommentar ordnet Looijenga (s.S. 134-135) diese Patienten in das Spektrum der Keimzelltumorgruppe unter Berücksichtigung von Alter, Histologie und Molekularbiologie ein, unter Hinweis auf die Embryogenese werden die unterschiedliche Tumorbiologie und das therapeutische Ansprechen plausibel erklärt.

Mit den drei Kategorien von Fallbeschreibungen unterstreicht die Klinische Pädiatrie die besondere Wertigkeit von Einzelfalldarstellungen für das gesamte Gebiet der Kinderheilkunde und Jugendmedizin [10]; durch die unterschiedlich langen Formate sollen die Beobachtungen akzentuiert und dem Leser die als wesentlich erachteten Informationen schneller verfügbar gemacht werden. Während die klassischen Fallberichte der Erweiterung des Krankheitsbildes in diagnostischer oder therapeutischer Sicht dienen, werden mit der Kategorie Pictorial Essay/Visite Fallberichte publiziert, deren Bildmaterial von besonderem edukativen Wert sind oder deren klinischer Verlauf außergewöhnlich ist. Kurzberichte enthalten eine kurze klare Botschaft für die klinische Praxis.

Die vorgestellten Arbeiten sind gleichermaßen Beispiele für die horizontale Vernetzung innerhalb des eigenen Fachgebietes wie auch mit anderen Fachdisziplinen und der klinischen Forschung, der sich die Klinische Pädiatrie in besonderer Weise verpflichtet fühlt [10]. Als Brücke zur Grundlagenforschung unter Hinweis auf gesicherte oder mögliche klinische Anknüpfungspunkte werden regelmäßig Übersichtarbeiten abgedruckt [8,9], die üblicherweise in klinisch orientierten Zeitschriften den aktuellen Wissensstand aus diagnostischer und therapeutischer Sicht unter dem Aspekt der Weiterbildung resümieren $[15,18]$ bzw. unter einem Leitthema wie z.B. Störungen der Geschlechtentwicklung [17] oder Schilddrüsenerkrankungen [11] umfassend mit Arbeiten aus unterschiedlichen Perspektiven abhandeln. Dagegen wird im Teil Berichte der Gesellschaft für Pädiatrische Hämatologie und Onkologie die vertikale Vernetzung innerhalb der pädiatrischen Onkologie besonders herausgestellt.

Brecht und Mitarbeiter (s.S. 181-185) stellen das neu gegründete GPOH-Rare-Tumor-Netzwerk als Internetplattform vor, durch die so unterschiedliche Kompetenzen wie die des behandelnden Arztes, von nationalen bzw. internationalen Registern und molekularbiologischen Speziallaboren miteinander verbunden werden. Zielvorstellung ist es, durch einheitliche Erfassung sowie Referenzbeurteilung den Ärzten und Patienten mit sehr seltenen Tumoren eine ähnlich auf Evidenz basierte Behandlung zu ermöglichen, wie dies bei Studienpatienten mit häufigeren Erkrankungen im Rahmen der etablierten Therapieoptimierungsstudien seit Jahrzehnten der Fall ist [5,25]. Da erwiesen ist, dass die Behandlungsaussichten für Patienten, die in einer spezialisierten Klinik im Rahmen einer klinischen Studie behandelt werden, besser sind als die für Nicht-Studienpatienten [22,23], ist zu erwarten, dass ein solches Netzwerk auch die Versorgung von Patienten mit seltenen Tumoren optimiert.

Im abschließenden Artikel stellen Kuhlen und Mitarbeiter (s.S. 186-193) das Düsseldorfer Modell einer ambulanten Palliativmedizin vor. Ihr Bericht beschreibt eindrücklich, wie aus zunächst unstrukturierten Einzelinitiativen ein systematisches Palliativkonzept erarbeitet wurde, dessen Honorierung zuletzt auch bei den Krankenkassen durchgesetzt werden konnte. Das entwickelte Konzept eignet sich als Modell für die Entwicklung weiterer Palliativverbünde nicht nur im Bereich der pädiatrischen Onkologie, sondern der gesamten Pädiatrie.

Andere Beispiele der horizontalen und vertikalen Vernetzung innerhalb der GPOH betreffen fächerübergreifend die Schnittstellen und selbstdefinierten Anforderungen der pädiatrischen 
Radioonkologie $[1,16]$, die psychosoziale Betreuung während der Behandlung [21], die Nachbeobachtung und Langzeitnachsorge sowie Spätfolgenerhebung im Verbund mit dem Kinderkrebsregister [4].

Die Vernetzung macht in anderen Teilbereichen der Kinder- und Jugendmedizin vergleichbar große Fortschritte, insbesondere der Neonatologie, wie einige Beispiele zeigen. So beinhalten die neuen Richtlinien für die Reanimation bei Früh- und Neugeborenen Vorschläge für ihre praktische Umsetzung [12]. Die Durchführung der Schmerz- und Pharmakotherapie $[14,19]$ sowie deren Kompatibilität bei intravenöser Verabreichung [13] wirken sich direkt auf die Behandlung aus. Studien zur Langzeitprognose sehr kleiner Frühgeborener [20] und ihr Anteil an Kindern mit Bedarf für sonderpädagogische Maßnahmen [24] führen zur Etablierung neuer Strukturen.

Die Nutzung von Vernetzungen im Einzelfall ist im G-DRG-System abzubilden, da die Berücksichtigung der Pathogenese zu einer begründeten und möglichst zielgerichteten Therapie bei dem individuellen Patienten führen soll. Dagegen sind für den Aufbau von klinischen diagnosebezogenen Registern, die Durchführung von Längsschnittuntersuchungen oder die Erstellung evidenzbasierter Leit- und Richtlinien zusätzliche Ressourcen bereitzustellen und einzuwerben. In besonderer Weise sind hier staatliche Modellmaßnahmen und gemeinnützige Förderungen zu nennen. Beispielsweise wären die Therapieoptimierungsstudien für krebskranke Patienten ohne die entsprechende Modellmaßnahme von 1978-1988 der Deutschen Bundesregierung nicht zustande gekommen [3], die in Folge von nichtstaatlichen gemeinnützigen Institutionen wie z. B. der Deutschen Krebshilfe als zeitlich limitierte Forschungsvorhaben weiter gefördert werden. Diese konzertierten Aktionen haben innerhalb von 25 Jahren die Langzeitüberlebensraten bei krebskranken Kindern und Jugendlichen ansteigen lassen, sodass heute drei von vier Kindern überleben [6]. Als weitere Modellmaßnahme haben die Ausschreibungen für Kompetenznetzwerke in der Medizin die horizontale und vertikale Vernetzung zum Inhalt [2]. Hier ist zu hoffen, dass die schon entstandenen und noch entstehenden Strukturen, die in nachhaltiger Weise zu Verbesserungen führen, durch andere Finanzierungsmodelle langfristig abgesichert werden.

Diese Aktivitäten (Clinical Practise) unter Wahrung wissenschaftlicher Kriterien sieht die Klinische Pädiatrie als gleichwichtige Aufgabe wie die klinische Forschung (Clinical Research) [10]. Trotz der zwischenzeitlichen Fortschritte bedarf es weiterer erheblicher Anstrengungen und Ressourcen, um das Ziel einer evidenzbasierten Medizin bei Kindern zu erreichen. Bei der relativen Seltenheit der meisten pädiatrischen Erkrankungen sind nationale und zunehmend internationale Studien erforderlich, für deren Durchführung das durch die Europäische Union im 7. Rahmenplan geförderte European Clinical Research Infrastructures Network (ECRIN) wichtige Mittlerfunktionen übernehmen kann [8].

\section{Literatur}

1 Bölling $T$ et al. Register zur Erfassung von Spätfolgen nach Strahlentherapie im Kindes- und Jugendalter - erste Ergebnisse. Klin Padiatr 2007; 219: 139-145

2 Bundesministerium für Bildung und Forschung. „Kompetenznetze in der Medizin“ Öffentliche Bekanntmachung: 1997, Förderzeitraum: 1999-2007, Gesamtvolumen: ca. 130 Mio. EUR, Geförderte Kompetenznetze: 9

3 Bundesministers für Forschung und Technologie. „Vergleichende Therapiestudien im Bereich bösartiger Neubildungen“ 1979-1989

4 Calaminus G, Kaatsch P. Positionspapier der Gesellschaft für Pädiatrische Onkologie und Hämatologie (GPOH) zu (Langzeit-)Nachbeobachtung, (Langzeit-)Nachsorge und Spätfolgenerhebung bei pädiatrisch-onkologischen Patienten. Klin Padiatr 2007; 219: 173-178

5 Creutzig $U$ et al. Krebserkrankungen bei Kindern: Erfolg durch einheitliche Therapiekonzepte seit 25 Jahren. Deutsches Ärzteblatt 2003; 100: A-842/B-712/C-665

6 Demotes-Mainard J, Ohmann C. European Clinical Research Infrastructures Network: promoting harmonisation and quality in European clinical research. Lancet 2005; 365 (9454): 107-108

7 Einhorn LH, Donohue JP. Improved chemotherapy in disseminatedtesticular cancer. J Urol 1977; 117: 65-71

8 Faber J, Armstrong SA. Defining leukemia stem cells in MLL-translocated leukemias: Implications for novel therapeutic strategies. Klin Padiatr 2007; 219: 306-311

9 Frühwaldt MC, Witt $O$. The epigenetics of cancer in children. Klin Padiatr 2008; 220: 333-341

10 Göbel U, Gortner L. Klinische Forschung und Behandlung in der Pädiatrie. Klin Padiatr 2008; 220: 2-4

11 Grüters A, Reinhardt D. (Red.) Schilddrüsenerkrankungen. Monatsschr Kinderheilkd 2008; 156: 949-995

12 Hansmann $G$ et al. Neue Reanimationsrichtlinien der ILCOR bei Frühund Reifgeborenen: Kritische Diskussion und Vorschläge zur praktischen Umsetzung. Klin Padiatr 2007; 219: 50-57

13 Höpner J-H et al. Erstellung einer Kompatibilitätstabelle für die intravenöse Pharmakotherapie auf neonatologischen und pädiatrischen Intensivstationen. Klin Padiatr 2007; 219: 37-43

14 Hünseler $C$, Roth $B$. Medikamentöse und nichtmedikamentöse Schmerztherapie in der Neonatologie: Zusammenstellung der aktuellen Datenlage. Klin Padiatr 2008; 220: 224-237

15 Koletzko B, Koletzko S. Gedeihstörung und Untergewicht. Monatsschr Kinderheilkd 2008; 156: 803-816

16 Kortmann et al. Anforderungen und Leistungsprofile der Arbeitsgemeinschaft Pädiatrische Radioonkologie (APRO): Eine Bestandsaufnahme und Darstellung zukünftiger Entwicklungen. Klin Padiatr 2007; 219: 166-172

17 Lentze MJ. (Red.) Störungen der Geschlechtsentwicklung. Monatsschr Kinderheilkd 2008; 156: 215-248

18 Lepach AC, Petermann F. Gedächtnisstörungen. Monatsschr Kinderheilkd 2007; 155: 753-763

19 Lindner $U$ et al. Drug utilisation in very preterm infants: Any changes during the past decade. Klin Padiatr 2008; 220: 238-242

20 Schlösser RL et al. Welchen Anteil haben ehemalige Frühgeborene an der Prävalenz von sonderpädagogischen Maßnahmen bei Schulkindern einer deutschen Großstadt (Frankfurt am Main)? Klin Padiatr 2008; 220: 57-60

21 Schreiber-Gollwitzer B et al. Positionspapier der GPOH und der DLFH zur psychosozialen Versorgung pädiatrisch-onkologischer Patienten in der Akutklinik. Klin Padiatr 2007; 219: 368-371

22 Stiller CA. Centralised treatment, entry to trials and survival. Br J Cancer 1994; 70: 352-362

23 Stiller CA, Eatock EM. Patterns of care and survival for children with acute lymphoblastic leukaemia diagnosed between 1980-94. Arch Dis Child 1999; 81: 202-208

24 Straßburg HM et al. Langzeitprognose sehr kleiner Frühgeborener in Deutschland. Klin Padiatr 2008; 220: 61-65

25 Thiele KP, Rheineberger P. „Unmöglich“ gibt es nicht. Deutsches Ärzteblatt 2003; 100: A1044-A1046 